\title{
Carbon nanodot-based electrogenerated chemiluminescence biosensor for miRNA-21 detection
}

\author{
Laura Gutiérrez-Gálvez ${ }^{1} \cdot$ Tania García-Mendiola $^{1,2,3}$. Cristina Gutiérrez-Sánchez ${ }^{1}$ (1) - Tamara Guerrero-Esteban ${ }^{1}$. \\ Cristina García-Diego ${ }^{4}$. Irene Buendía ${ }^{5} \cdot$ M. Laura García-Bermejo ${ }^{5}$. Félix Pariente ${ }^{1,2} \cdot$ Encarnación Lorenzo $^{1,2,3}$
}

Received: 31 May 2021 / Accepted: 25 September 2021 / Published online: 30 October 2021

(c) The Author(s) 2021, corrected publication 2022

\begin{abstract}
A simple carbon nanodot-based electrogenerated chemiluminescence biosensor is described for sensitive and selective detection of microRNA-21 (miRNA-21), a biomarker of several pathologies including cardiovascular diseases (CVDs). The photoluminescent carbon nanodots (CNDs) were obtained using a new synthesis method, simply by treating tiger nut milk in a microwave reactor. The synthesis is environmentally friendly, simple, and efficient. The optical properties and morphological characteristics of the CNDs were exhaustively investigated, confirming that they have oxygen and nitrogen functional groups on their surfaces and exhibit excitation-dependent fluorescence emission, as well as photostability. They act as co-reactant agents in the anodic electrochemiluminescence $(\mathrm{ECL})$ of $\left[\mathrm{Ru}(\mathrm{bpy})_{3}\right]^{2+}$, producing different signals for the probe (single-stranded DNA) and the hybridized target (double-stranded DNA). These results paved the way for the development of a sensitive ECL biosensor for the detection of miRNA-21. This was developed by immobilization of a thiolated oligonucleotide, fully complementary to the miRNA-21 sequence, on the disposable gold electrode. The target miRNA-21 was hybridized with the probe on the electrode surface, and the hybridization was detected by the enhancement of the $\left[\mathrm{Ru}(\mathrm{bpy})_{3}\right]^{2+} / \mathrm{DNA}$ ECL signal using CNDs. The biosensor shows a linear response to miRNA-21 concentration up to $100.0 \mathrm{pM}$ with a detection limit of $0.721 \mathrm{fM}$. The method does not require complex labeling steps, and has a rapid response. It was successfully used to detect miRNA-21 directly in serum samples from heart failure patients without previous RNA extraction neither amplification process.
\end{abstract}

Keywords Green chemistry synthesis $\cdot$ Carbon nanomaterials $\cdot$ RNA detection $\cdot$ ECL biosensor

Tania García-Mendiola

tania.garcia@uam.es

$\triangle$ Cristina Gutiérrez-Sánchez

cristina.gutierrezs@uam.es

1 Department of Analytical Chemistry and Instrumental Analysis, Universidad Autónoma de Madrid, Ciudad Universitaria de Cantoblanco, 28049 Madrid, Spain

2 Institute for Advanced Research in Chemical Sciences (IAdChem), Universidad Autónoma de Madrid, Ciudad Universitaria de Cantoblanco, 28049 Madrid, Spain

3 IMDEA Nanociencia, Ciudad Universitaria de Cantoblanco, 28049 Madrid, Spain

4 Instituto de Catálisis y Petroleoquímica, Consejo Superior de Investigaciones Científicas, C/Marie Curie 2, 28049 Madrid, Spain

5 Biomarkers and Therapeutic Targets Group and Core Facility, Instituto Ramón y Cajal de Investigación Sanitaria (IRYCIS), Spanish Renal Research Network (REDinREN), Madrid, Spain

\section{Introduction}

Carbon nanomaterials are currently of great interest for their potential applications in different fields [1-3]. This includes the recent use of a new class of fluorescent carbon nanomaterials called carbon nanodots (CNDs). These carbon-based nanomaterials have excellent properties: in particular, they have good water solubility and low toxicity, as well as high chemical stability. Hence, unlike semiconductor quantum dots $[4,5]$, these nanomaterials are biocompatible and not harmful to the environment. In recent years, CNDs have been actively investigated and widely used in interesting applications such as biosensing $[6,7]$. In this context, they have been combined with electrochemiluminescence, leading to a new range of possibilities in the field of biosensing [8-10].

Electrogenerated chemiluminescence or electrochemiluminescence (ECL) is the generation of light occurring at the 
electrode when suitable molecules or ions are electrochemically activated and undergo highly exergonic electron-transfer reactions to form excited-state species (luminophores or emitters) that are capable of causing luminescence [11]. The mechanisms associated with these phenomena are well known and have been described in the literature, being classified into two main types [12,13], the annihilation pathway and the co-reactant pathway. The latter is especially useful when charged radical ions are not stable enough for the ECL annihilation reaction or when one of the radical ions cannot be formed because the solvent has a narrow potential window.

The energy from the excitation source in ECL assays has a different nature than the signal, compared to fluorescence, giving ECL certain advantages such as a high signal to noise ratio. It therefore has great potential as a detection system for (bio)sensors [14, 15]. ECL-based biosensors have demonstrated remarkable properties and advantages such as controllability, high sensitivity, and low background noise. In fact, DNA sensing based on the detection of hybridization by ECL has drawn much attention [16-19]. Usually, the DNA acts as a co-reactant [20-24]. However, most of these sensors are not very sensitive.

Currently, substantial efforts are being made to improve the performance of these devices by exploring new materials and approaches with high sensitivity, high stability, and excellent controllability. In this sense, the use of CNDs opens new possibilities for the biosensor field, due to the optoelectronic properties of these nanomaterials. However, although some studies have described the use of CNDs as nanoemitters $[25,26]$ or co-reactants for the anodic ECL of tris $\left(2,2^{\prime}\right.$-bipyridyl)dichlororuthenium(II) $\left(\left[\mathrm{Ru}(\mathrm{bpy})_{3}\right]^{2+}\right)$ [27], the most widely used luminophore in this technique much remains to be explored in this regard.

MicroRNAs (miRNAs) are an important class of small non-coding RNA involved in the regulation of gene expression and whose mature products are around $18-25$ nucleotides long. Their dysregulation has been associated with Alzheimer's disease, cardiovascular diseases, and different types of cancer [28]. Thus, miRNAs can be used as biomarkers for early diagnosis and prognosis of these diseases. In particular, miRNAs are attractive biomarkers of response to cardiovascular diseases therapy given their stability, tissue-specific expression patterns, and secretion into bodily fluids [29]. For example, miRNA-21 levels were increased in fibroblasts in the failing heart [30, 31]. According to World Health Organization reports, cardiovascular diseases (CVDs) are almost the major causes of death globally and are responsible for over 18 million deaths every year. Therefore, diagnostic and prognostic biomarkers as miRNA-21 for CVDs have attracted significant interest in recent years [32]. Hence, over the past few decades, various miRNA sensing methods based on different techniques have been reported, such as optical [33], electrochemical [34], microfluidic [35], and ICP-MS [36]. However, detection and quantification of miRNA-21 using existing methods are relatively laborintensive and time-consuming. Thus, the development of new rapid, simple, and effective methodologies for miRNA21 detection is an area of great interest and the scientific community needs to put more emphasis on improving the early diagnosis methods. As consequence, nowadays, a great number of research works are focused on the development of new methodologies for rapid biomarker detection and recently several detection methods of miRNA employing new nanomaterials as CNDs, or graphene quantum dots have been reported [37-40]. In this sense, the combination of CNDs with ECL can be an interesting approach [41, 42] since these CNDs are employed to replace the traditional quantum dots as electrochemiluminescent luminophores. In addition to the simplicity of its synthesis and not requiring contaminating reagents, it presents advantages in terms of the analytical characteristics of the biosensor developed using this nanomaterial. In particular, for mRNA sensing, a low detection limit is achieved without the need of coupling more complicated methods, such as the isothermal circular strand-replacement polymerization (CSRP).

In this article, we describe a new sensitive method for the direct detection of miRNA-21. It consists of a new ECL biosensor based on water-soluble CNDs. We describe the synthesis of the CNDs, following environmentally friendly procedures, and their application as co-reactants to develop the ECL biosensor. Although some sensitive ECL biosensors for miRNA have previously been reported [43-46], they require complex and time-consuming development and labeling steps. Therefore, the search for new and quick methods for determining this biomarker is of great interest. Moreover, we do not use toxic reagents or time-consuming purification steps for the synthesis of these CNDs. Instead, we use natural products and non-toxic solvents.

The proposed biosensor does not require a complex procedure for its manufacture or complex labeling steps, as shown in Scheme 1. Briefly, the thiolated capture probe (miRNA-21-SH) is deposited on a disposable gold screenprinted electrode (AuSPE). Next, the analyte miRNA-21 is hybridized with the capture probe on the electrode surface. Finally, the hybridization is detected by the enhanced ECL emission of the $\left[\mathrm{Ru}(\mathrm{bpy})_{3}\right]^{2+} / \mathrm{CNDs}$ system, allowing reliable sensing of miRNA-21. The method we have developed is highly sensitive, allowing fast, direct, and simple trace biomarker detection directly in serum samples from heart failure patients without previous RNA extraction neither amplification process. 

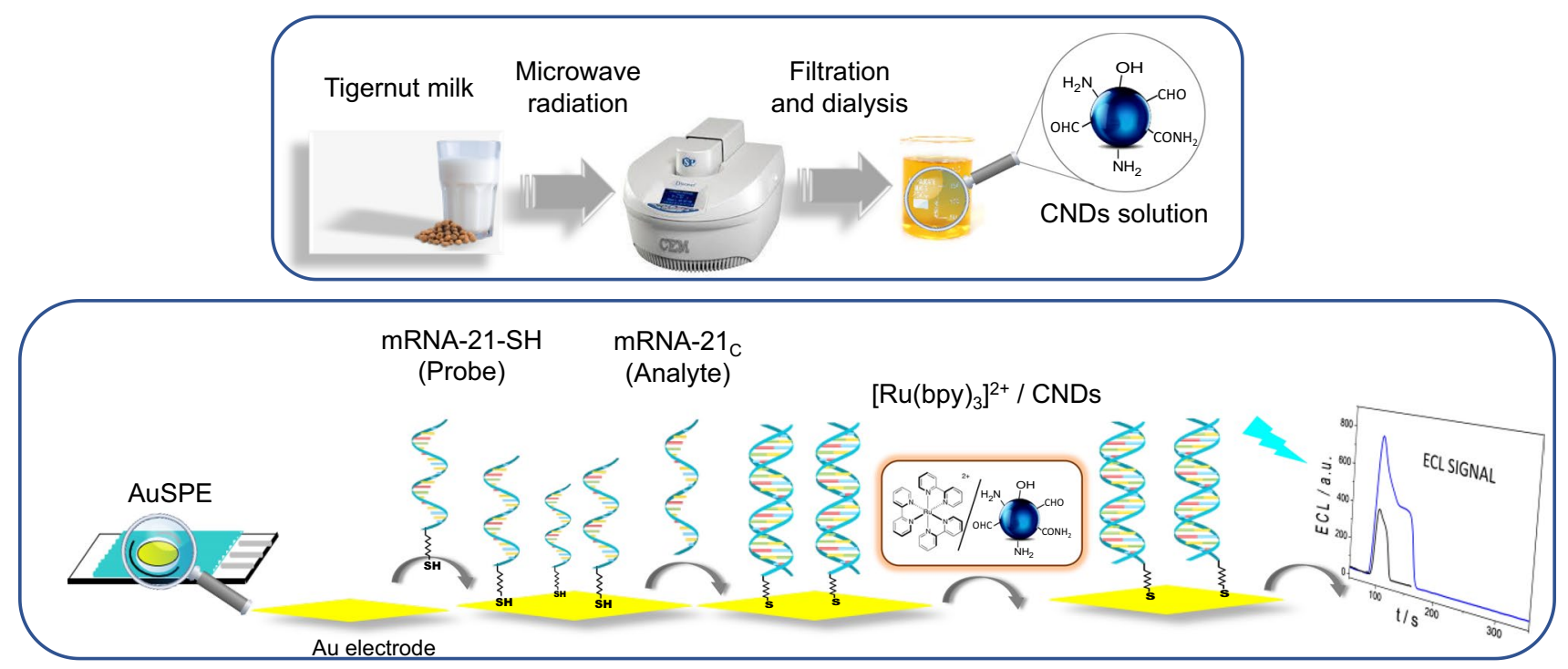

Scheme 1 Steps of the friendly environmental chemistry procedure for CNDs synthesis and schematic representation of the DNA biosensor development: probe immobilization, hybridization with the analyte and ECL detection using $\left[\mathrm{Ru}(\mathrm{bpy})_{3}\right]^{2+} / \mathrm{CNDs}$ system

\section{Experimental}

\section{Chemicals}

Tiger nut milk (from a local supermarket) was used as a natural precursor for the CNDs. Monobasic sodium phosphate $\left(\mathrm{NaH}_{2} \mathrm{PO}_{4} \cdot \mathrm{H}_{2} \mathrm{O}\right)$, dibasic sodium phosphate $\left(\mathrm{Na}_{2} \mathrm{HPO}_{4} \cdot 2 \mathrm{H}_{2} \mathrm{O}\right)$, sodium chloride $(\mathrm{NaCl})$, hydrochloric acid $(\mathrm{HCl})$, sodium hydroxide $(\mathrm{NaOH})$, sulfuric acid $\left(\mathrm{H}_{2} \mathrm{SO}_{4}\right)$, tris $\left(2,2^{\prime}\right.$-bipyridyl) dichlororuthenium (II) hexahydrate $\left(\left[\mathrm{Ru}(\mathrm{bpy})_{3}\right] \mathrm{Cl}_{2} \cdot 6 \mathrm{H}_{2} \mathrm{O}\right)$, and human serum (from human male AB plasma, ref. H4522) were supplied by Merck (https://www.merckgroup.com/).

miRNeasy Serum/Plasma Advanced Kit (50), RNA Spike-in kit, UniRT, SYBR® Green master mix, UniSp2, LNA control primer set, UniRT, hsa-miR-103a-3p miRCURY LNA miRNA PCR Assay.cel-miR-39-3p miRCURY LNA miRNA PCR Assay and hsa-miR-21-5p LNA ${ }^{\mathrm{TM}}$ PCR primer set, UniRT were supplied by Qiagen (https://www. qiagen.com).

\section{Synthetic DNA oligonucleotides}

Synthetic 22-mer oligonucleotides from Merck (https:// www.merckgroup.com/) were used. A single-stranded DNA sequence modified at the $5^{\prime}$ end with a hexalkyl thiol was used as the capture probe, denoted miRNA-21-SH. For target analytes, a fully complementary sequence (denoted miRNA- $21_{C}$ ) and a non-complementary sequence (denoted miRNA-21 $1_{\mathrm{NC}}$ ) were used. All sequences are listed in Table 1.

Stock solutions of the oligonucleotides corresponding to the complementary $\left(\right.$ miRNA- $21_{\mathrm{C}}$ ), non-complementary
Table 1 Synthetic oligonucleotide sequences used

\begin{tabular}{ll}
\hline Nomenclature & Oligonucleotide sequence \\
\hline miRNA-21-SH & 5'-SH- $\left(\mathrm{CH}_{2}\right)_{6}$-TCAACATCAGTCTGATAAGCTA \\
miRNA-21 $_{\mathrm{C}}$ & 5'-UAGCUUAUCAGACUGAUGUUGA \\
miRNA-21 $_{\mathrm{NC}}$ & 5'-AUCGAAUAGUCUGACUACAACU \\
miRNA-21 $_{\mathrm{SM}}$ & 5'-UAGCUUAUCTGACUGAUGUUGA \\
miRNA-144 & 5'-UAACACUGUCUGGUAAAGAUGG \\
miRNA-155 & 5'-UUAAUGCUAAUCGUGAUAGGGGU
\end{tabular}

(miRNA-2 $1_{\mathrm{NC}}$ ), and single-mismatched (miRNA-21 $1_{\mathrm{SM}}$ ) sequences were prepared at a concentration of $100 \mu \mathrm{M}$ in $10 \mathrm{mM}$ phosphate buffer (PB) with $0.4 \mathrm{M} \mathrm{NaCl}, \mathrm{pH} 7.0$. Prior to use, thiol-modified probes were treated with DTT and then purified by elution through a NAP-10 column of Sephadex G-25. Afterwards, the stock solution of the thiolated miRNA-21-SH probe was prepared at a concentration of $9.00 \mu \mathrm{M}$ in $10 \mathrm{mM} \mathrm{PB}, \mathrm{pH} 7.0$. Aliquots of a few microliters of all stock solutions were stored at $-20^{\circ} \mathrm{C}$.

\section{miRNA-21 serum samples from heart failure patients}

miRNA-21 serum samples from heart failure patients were provided by IRICYS (Instituto Ramón y Cajal de Investigación Sanitaria) from the Biobank collection and correspond to the clinical study approved by the Local Ethics Committee (references 175/13 and 061/16) and complied with the Declaration of Helsinki. Serum clinical sample was obtained from whole blood samples by centrifugation, and aliquots of $250 \mu \mathrm{L}$ were stored at $-80{ }^{\circ} \mathrm{C}$. Two miRNA-21 
serum clinical samples were used: serum 1 from heart failure patient with high levels of miRNA-21 expressed and serum 2 with low levels of miRNA-2, estimated by qRT-PCR at Ramon y Cajal Institute.

\section{Microscopic and spectroscopic techniques}

The transmission electron microscope used was a JEOL JEM $2100 \mathrm{HT}$ operating at $200 \mathrm{kV}$. Images were processed using the Fiji software.

LECO CHNS-932 elemental analysis equipment and a FTIR (Fourier-transform infrared) Bruker IFS60v spectrophotometer were used.

UV-visible spectra were recorded from 200 to $800 \mathrm{~nm}$ in $1.0 \mathrm{~cm}$ quartz cells in a double-beam PharmaSpec UV-1700 series spectrophotometer from Shimadzu Corporation.

For fluorescence measurements, a Varian Cary Eclipse spectrofluorometer and quartz cuvettes with a $1-\mathrm{cm}$ optical path were used.

X-ray photoelectron spectra (XPS spectra) of CNDs sample were obtained using a PHOIBOS 150 9MCD spectrometer (SPECS $\mathrm{GmbH}$ ) equipped with a monochromatic $\mathrm{Al} \mathrm{K \alpha}$ $\mathrm{X}$-ray source operating at $200 \mathrm{~W}$ and $12 \mathrm{kV}(1486.7 \mathrm{eV})$. Pass energies of 50 and $20 \mathrm{eV}$ were used for acquiring both survey and high-resolution spectra, respectively. Survey data were acquired with an energy step of $1 \mathrm{eV}$ and $100 \mathrm{~ms}$ dwell time per point. The high-resolu

tion scans were taken around the emission lines of interest with $0.1 \mathrm{eV}$ steps and $100 \mathrm{~ms}$ dwell time per point.

Fluorescent microscopic images were taken on a Zeiss Axiovert 200 inverted microscope with a monochrome CCD camera and the Fiji software. Lumencor's SPECTRA-X light engine was used. A $10 \times / 0.45$ Plan-Apochromat Ph1 objective lens was used for detection. A DAPI filter (395/25) was employed, recording emissions with a DAPI filter (432/36).

Zeta potential measurements were determined at $25^{\circ} \mathrm{C}$ using a Zetasizer Nano ZS instrument (Malvern Instrument Ltd., Grovewood, Worcestershire, UK).

\section{Electrochemical and electrochemiluminescence equipment}

For electrochemical measurements, we used an Autolab/ PGSTAT 10 potentiostat from Eco Chemie with the GPES 4.9 software. For electrochemiluminescence (ECL), a Metrohm DropSens ECL equipment was used.

Integrated screen-printed gold electrodes $(4 \mathrm{~mm}$ diameter, AuSPEs) from DropSens S.L (Oviedo, Spain) were used as working electrodes. The format of these screen-printed electrodes includes a gold working electrode, a silver pseudo reference electrode, and a gold counter electrode. The electrodes were connected using a SPE Connector (DropSens S. L.) as interface. Sixty microliters of the sample was deposited onto the electrochemical cell to carrying out the electrochemical experiments ("drop test"). The electrochemical cell has not been degassed before carrying out the electrochemical experiments.

All material and solutions were sterilized in a Nüve OT012 autoclave before use.

\section{Procedures}

\section{Synthesis of the CNDs}

In a typical synthesis, $3 \mathrm{~mL}$ of tiger nut milk was heated in a quartz flask under magnetic stirring for $30 \mathrm{~min}$ using a microwave reactor. The temperature and time were controlled and adjusted to maintain a constant power of $150 \mathrm{~W}$ and pressure of $170 \mathrm{psi}$ [47]. These conditions produced yellow solutions. The solutions obtained were allowed to cool to room temperature. Next, they were filtered with a $0.45 \mu \mathrm{m}$ nylon syringe filter and dialyzed with a Spectra/ Por® 6 membrane (MWCO, $1 \mathrm{KDa}$ ) for one and a half hours to purify the synthesized samples. Finally, they were stored protected from light.

\section{Preparation of microscope samples}

The samples used for transmission electron microscopy (TEM) were prepared by depositing a $400 \mu \mathrm{M}$ CNDs solution onto a carbon-film grid for direct observation after drying. To visualize the CNDs under the fluorescence microscope, $20 \mu \mathrm{L}$ of a $40 \mu \mathrm{M}$ solution of CNDs was placed on a slide and allowed to dry for $48 \mathrm{~h}$.

\section{Electrochemical pre-treatment of the electrode}

AuSPEs were activated by a mild electrochemical pre-treatment that consisted of immersing them in a $0.5 \mathrm{M} \mathrm{H}_{2} \mathrm{SO}_{4}$ solution and applying ten successive cyclic potential sweeps at $0.1 \mathrm{~V} / \mathrm{s}$ between -0.30 and $+1.30 \mathrm{~V}$.

\section{Biosensor development}

Biosensor development consists of three fundamental stages, as shown in Scheme 1, DNA capture probe immobilization, hybridization, and ECL detection.

Immobilization of the miRNA-21-SH probe by chemisorption of thiols Ten microliters of the $9.00 \mu \mathrm{M}$ solution of miRNA21-SH in $10 \mathrm{mM}$ PB was deposited onto the surfaces of clean, activated gold electrodes. After $72 \mathrm{~h}$ to complete evaporation, the modified electrodes were briefly immersed in purified water to remove non-adsorbed material [48]. 
Hybridization of the miRNA-21-SH probe with the miRNA-21 Ten microliters of a miRNA-21c solution (from $2.34 \mathrm{fM}$ to $100.0 \mathrm{pM}$ ) was added onto the electrode surface modified with the thiolated miRNA-21-SH probe. Next, they were kept in a humid chamber at $40{ }^{\circ} \mathrm{C}$ for $1 \mathrm{~h}$. Finally, they were briefly immersed in purified water to remove nonadsorbed material.

Electrochemiluminescent detection Electrochemiluminescent measurements were performed by depositing $60.0 \mu \mathrm{L}$ of $7 \mathrm{mM}\left[\mathrm{Ru}(\mathrm{bpy})_{3}\right]^{2+}$ in $0.2 \mathrm{M} \mathrm{PB}, \mathrm{pH} 8.0$, with or without $70 \mu \mathrm{M}$ CNDs, on the electrode surface modified with the hybridized miRNA samples.

To produce the ECL reaction, cyclic potential sweeps from 0.0 to $1.15 \mathrm{~V}$ for $\left[\mathrm{Ru}(\mathrm{bpy})_{3}\right]^{2+}$ and from 0.0 to $1.30 \mathrm{~V}$ for $\left[\mathrm{Ru}(\mathrm{bpy})_{3}\right]^{2+} / \mathrm{CNDs}$ were performed with a potential step of $0.002 \mathrm{~V}$ and a scan rate of $10 \mathrm{mV} / \mathrm{s}$. Cyclic voltammograms and ECL spectra were recorded simultaneously.

\section{miRNA-21 determination in spiked human serum samples}

miRNA-21 was determined in human serum samples spiked with miRNA-21c to assess the applicability of the biosensor developed. Firstly, human serum spiked with miRNA-21c was prepared at a final concentration of $1.00 \mathrm{pM}$ miRNA-21c (2.0 $\mu \mathrm{L}$ of $10.0 \mathrm{pM}$ miRNA-21c solution was taken to a final volume of $20 \mu \mathrm{L}$ with human serum). Then, $10.0 \mu \mathrm{L}$ of this solution was added to the electrode surface modified with the thiolated miRNA21-SH probe. Next, the hybridization process described above (in a humid chamber at $40{ }^{\circ} \mathrm{C}$ for $1 \mathrm{~h}$ ) is developed. Finally, the electrodes were briefly immersed in purified water to remove non-adsorbed material. After this, ECL measurements were performed as described above, using a mixture of $7 \mathrm{mM}\left[\mathrm{Ru}(\mathrm{bpy})_{3}\right]^{2+}$ and $70 \mu \mathrm{M}$ CNDs in $0.2 \mathrm{M} \mathrm{PB}$ pH 8.0. Finally, the miRNA-21c concentration was estimated using the ECL average value obtained for the spiked human serum and the calibration plot $\left(\mathrm{ECL}=29.1 \log \left[\right.\right.$ miRNA-21 $\left.\left._{\mathrm{C}}\right]+722.3\right)(R=0.9996)$, using the external calibration method, but subtracting the corresponding signal of the blank (the ECL signal of the diluted human serum without miRNA-21).

\section{miRNA-21 determination in serum samples from heart failure patients}

miRNA-21 was detected in serum samples from heart failure patients. Experimental procedures are described in detail in Electronic Supplementary Material (ESM).

\section{Results and discussion}

\section{Choice of materials}

Carbon nanodots (CNDs) have been chosen for the development of efficient DNA ECL biosensors, due to their biocompatibility and their property to act as co-reactants for the anodic ECL of $\left[\mathrm{Ru}(\mathrm{bpy})_{3}\right]^{2+}[27]$.

\section{Synthesis and characterization of carbon nanodots}

Photoluminescent CNDs were synthesized by carbonization of tiger nut milk using a microwave reactor synthesizer, as shown in Scheme 1 and explained in detail in the "Experimental" section. The temperature and reaction time were optimized using emissions from synthesized CNDs (see Fig. S1). Best results, considering the balance of speed and performance, were obtained with a reaction time of $30 \mathrm{~min}$ at a temperature of $200{ }^{\circ} \mathrm{C}$, maintaining a constant pressure of $170 \mathrm{psi}$ and a power of $150 \mathrm{~W}$.

Hydrothermal treatments usually require carbonization for up to $18 \mathrm{~h} \mathrm{[49].} \mathrm{However,} \mathrm{only} 30 \mathrm{~min}$ is required with microwave radiation. Increasing the time of exposure to microwave radiation produces an increase in the emission intensity of the CNDs, which is more evident between 5 and 30 min (Fig. S1B). After 30 min, emissions remain almost constant, so this has been selected as the optimal synthesis time in terms of the fluorescence of the resulting product and the speed of the process.

The obtained CNDs were characterized by different techniques to elucidate their morphology, composition, and properties. Elemental analysis revealed that the CNDs contain $47.19 \%$ C, $5.75 \%$ H, $1.05 \%$ N (see Fig. S2), and 46.01\% $\mathrm{O}$ (calculated). Zeta potential measurements gave an average value in aqueous solution of $-6.7 \pm 0.3 \mathrm{mV}$. This negative value of zeta potential is probably due to the surface-contained carboxylate groups on the CNDs.

\section{Microscopic characterization}

The morphology and dimensions of the CNDs were determined by TEM. As shown in Fig. 1A, CNDs have a quasispherical morphology with diameters distributed in a range from 4 to $9 \mathrm{~nm}$ and an average size of $6.8 \pm 1.5 \mathrm{~nm}(n=35)$ (see histogram of Fig. S3).

\section{Spectroscopic characterization}

The UV-visible molecular absorption spectrum of the CNDs is shown in Fig. 1B. It can be seen that the CNDs have an absorption band at $284 \mathrm{~nm}$ that can be assigned to $n \rightarrow \pi^{*}$ 
Fig. 1 (A) TEM image of the synthesized CNDs. Inset: magnification of a CNDs TEM image. (B) Absorbance (black line) and fluorescence emission (blue line) spectra from 200 to $800 \mathrm{~nm}$, the latter exciting at the maximum of excitation peak $(\Lambda=380 \mathrm{~nm})$. (C) Fluorescence micrograph and (D) FT-IR spectrum of the synthesized CNDs
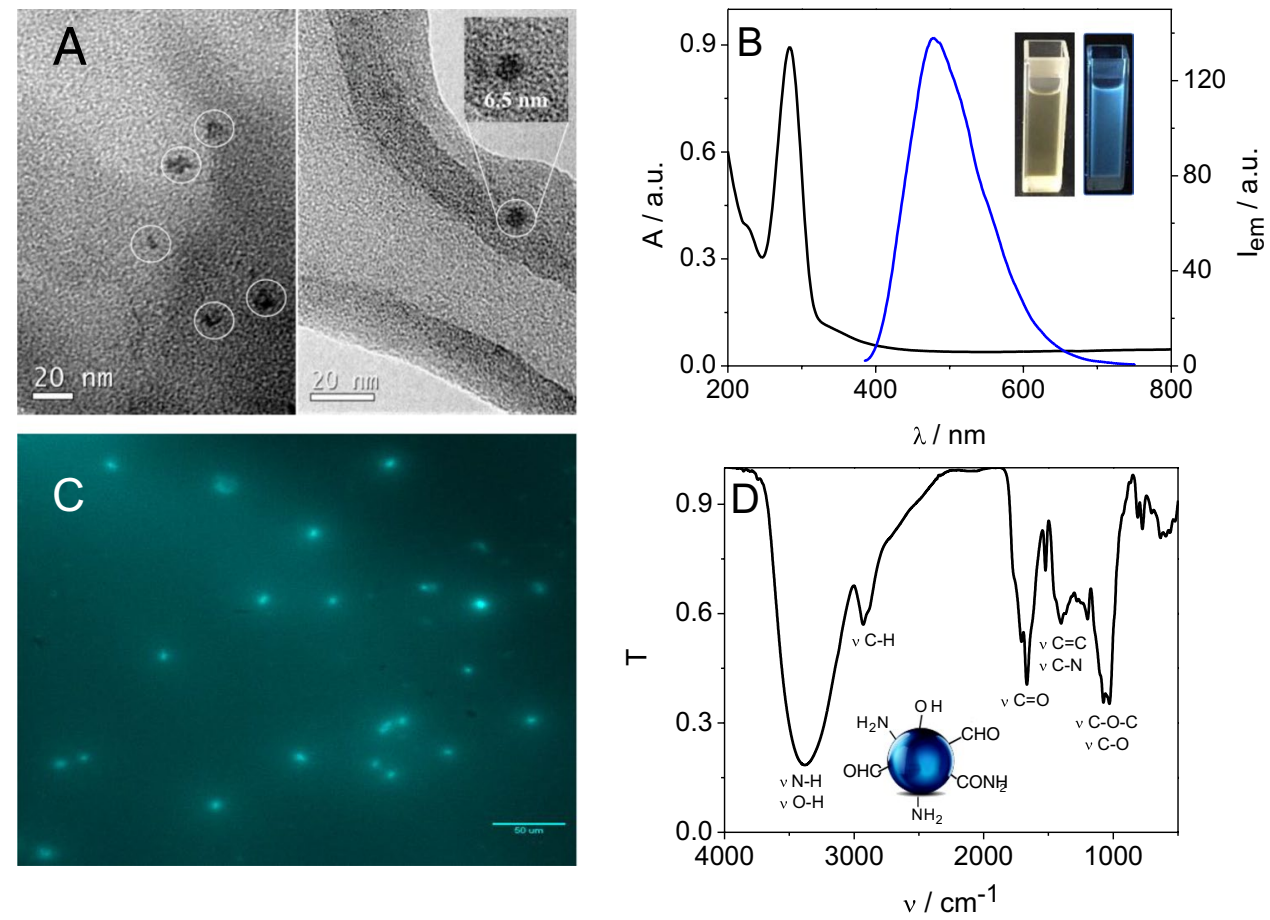

transitions from $\mathrm{C}=\mathrm{O}$ groups and $\pi \rightarrow \pi^{*}$ transitions from $\mathrm{C}=\mathrm{C}$ groups [50]. This feature is characteristic of CNDs synthesized by carbonization of carbon-based materials [51] and demonstrates the presence of carbonyl groups on the surface of the synthesized CNDs. The fluorescence emission spectrum recorded by exciting CNDs at the maximum excitation peak $(380 \mathrm{~nm})$ presents a maximum at $478 \mathrm{~nm}$ (Fig. 1B). The emission peak decreases and shifts its position to a higher emission wavelength (from 471 to $550 \mathrm{~nm}$ ) as $\lambda_{\mathrm{ex}}$ moves from 300 to $500 \mathrm{~nm}\left(\Delta \lambda_{\mathrm{ex}}=200 \mathrm{~nm}\right.$ ) (see Fig. S4). The redshift of the fluorescence emission can be attributed to differences in particle sizes and in the distribution of emissive sites on the surfaces of the CNDs. Smaller particles would be excited at shorter wavelengths than larger ones [52]. The fluorescence micrograph (Fig. 1C) again demonstrates the fluorescence of the CNDs, which appear as light spots.

The high carbon and oxygen content suggests that the particles obtained are nanometer-sized carbonaceous materials with a large number of carboxylic groups on the surface [49], a fact that can be confirmed by the FTIR spectrum (Fig. 1D). As can be seen, the spectrum exhibits the characteristic stretching band of $\mathrm{OH}$ and $\mathrm{NH}$ vibrations at 2900 and $3400 \mathrm{~cm}^{-1}$; a band located at $1650 \mathrm{~cm}^{-1}$ and attributed to $\mathrm{C}=\mathrm{O}$ stretching vibration confirms that $-\mathrm{COOH}$ groups are present, explaining the high-water solubility of the CNDs. The band present at $2900 \mathrm{~cm}^{-1}$ can be assigned to $\mathrm{C}-\mathrm{H}$ tension vibrations, and those above $1050 \mathrm{~cm}^{-1}$ are assigned to $\mathrm{C}-\mathrm{O}$ and $\mathrm{C}-\mathrm{O}-\mathrm{C}$ bonds. The band at $1400 \mathrm{~cm}^{-1}$ probably corresponds to $\mathrm{C}-\mathrm{N}$ or $\mathrm{C}=\mathrm{C}$ vibrations. Therefore, carbonyl groups, alcohols, and amines are present in the synthesized CNDs.

X-ray photoelectron spectroscopy (XPS) studies were carried out to corroborate the results obtained from FTIR spectroscopy. The results revealed the presence of carbon, oxygen, nitrogen, phosphorus, and sodium, with the following percentage atomic concentrations: $64.4 \% \mathrm{C}, 31.3 \% \mathrm{O}$, $3.9 \% \mathrm{~N}, 0.2 \% \mathrm{P}$, and $0.2 \% \mathrm{Na}$ (Fig. S5A). Peak fitting of the $\mathrm{C} 1 \mathrm{~s}$ core level region (Fig. $\mathrm{S} 5 \mathrm{~B}$ ) showed peaks at $\mathrm{C}=\mathrm{C}$ (284.3 eV), C-C/C-H (284.8 eV), C-O/C-N (286.0 eV), $\mathrm{C}=\mathrm{O}(287.6 \mathrm{eV}), \mathrm{O}-\mathrm{C}=\mathrm{O}(288.8 \mathrm{eV})$, and the characteristic $\pi \rightarrow \pi^{*}$ vibrations of carbon atoms in graphene-like structures $(292.4 \mathrm{eV})$. The O1s line fitting for the CNDs (Fig. S5C) shows two different chemical components centered at $531.5 \mathrm{eV}(\mathrm{O}=\mathrm{C})$ and $532.5 \mathrm{eV}(\mathrm{O}-\mathrm{C})$. The N1s spectrum (Fig. S5D) shows two peaks at $399.9 \mathrm{eV}$ and $401.5 \mathrm{eV}$, which were attributed to the presence of $\mathrm{C}-\mathrm{N}$ and $\mathrm{N}-\mathrm{H}$, respectively. The CND surface components determined by X-ray photoelectron spectroscopy are in good agreement with FTIR results.

The stability of the CNDs in aqueous solution was also studied following their fluorescence spectra. The results show that emission intensity remains constant for at least 30 days and then begins to decrease significantly (Fig. S6). We have also checked the reproducibility of the CND synthesis method. For this, the synthesis procedure has been repeated several times and no significant differences have been observed in the corresponding batches obtained, other than small differences in the concentration of CNDs. As we describe in detail in the "Electronic supplementary.material 
(ESM)" section, from the results obtained by elemental analysis, TEM, and UV-visible spectroscopy, we estimated the concentration of the synthesized CNDs. It was found to be of $400 \mu \mathrm{M}$, using the calculated molar extinction coefficient of $1.82 \times 10^{6} \mathrm{M}^{-1} \mathrm{~cm}^{-1}$ (see ESM and Fig. S7).

\section{Electrochemical characterization}

CNDs have been previously used as co-reactants in the anodic ECL of $\left[\mathrm{Ru}(\mathrm{bpy})_{3}\right]^{2+}[27,53]$. To evaluate whether these synthesized CNDs could act as co-reactants in the anodic ECL of $\left[\mathrm{Ru}(\mathrm{bpy})_{3}\right]^{2+}$, we recorded cyclic voltammograms of the CNDs, $\left[\mathrm{Ru}(\mathrm{bpy})_{3}\right]^{2+}$, and $\left[\mathrm{Ru}(\mathrm{bpy})_{3}\right]^{2+} /$ CNDs in 0.2 M PB, pH 8.0, at an AuSPE. As can be seen in Fig. 2A, $\left[\mathrm{Ru}(\mathrm{bpy})_{3}\right]^{2+}$ shows the characteristic redox process ascribed to the $\left[\mathrm{Ru}(\mathrm{bpy})_{3}\right]^{3+} /\left[\mathrm{Ru}(\mathrm{bpy})_{3}\right]^{2+}$ system, and the CNDs show no voltammperometric peaks within the potential window of the experiment. However, in presence of both CNDs and $\left[\mathrm{Ru}(\mathrm{bpy})_{3}\right]^{2+}$, there is an electrocatalytic process, since the intensity of the $\left[\mathrm{Ru}(\mathrm{bpy})_{3}\right]^{2+}$ oxidation peak increases concomitantly as the cathodic peak disappears; this was more evident on increasing the CND concentration from 40 to $70 \mu \mathrm{M}(40,60$, and $70 \mu \mathrm{M})$ when a fixed concentration of $7.0 \mathrm{mM}$ of $\left[\mathrm{Ru}(\mathrm{bpy})_{3}\right]^{2+}$ was used.

Figure $2 \mathrm{~B}$ shows that the ECL response of the $\left[\mathrm{Ru}(\mathrm{bpy})_{3}\right]^{2+} / \mathrm{CNDs}$ mixture produced a strong ECL signal, which is consistent with that observed by cyclic voltammetry, whereas $\left[\mathrm{Ru}(\mathrm{bpy})_{3}\right]^{2+}$ or CNDs alone exhibited weak ECL signals. Moreover, as observed by cyclic voltammetry, the ECL $\left[\mathrm{Ru}(\mathrm{bpy})_{3}\right]^{2+}$ signal increased on increasing the concentration of CNDs.

We believe that CNDs act as co-reactants in the $\left[\mathrm{Ru}(\mathrm{bpy})_{3}\right]^{2+} / \mathrm{CNDs}$ ECL system, being converted into reductive intermediates by the chemical oxidation of oxygen-containing units present on their surfaces by electrogenerated $\left[\mathrm{Ru}(\mathrm{bpy})_{3}\right]^{3+}[27]$, similar to the role played by TPrA in the anodic ECL of the $\left[\mathrm{Ru}(\mathrm{bpy})_{3}\right]^{2+} /$ TPrA system [34]. Therefore, we propose the following typical "oxidative-reductive" co-reactant pathway mechanism for the $\left[\mathrm{Ru}(\mathrm{bpy})_{3}\right]^{2+} / \mathrm{CNDs}$ ECL system:

$$
\begin{aligned}
& {\left[\mathrm{Ru}(\mathrm{bpy})_{3}\right]^{2+}-\mathrm{e}-\rightarrow\left[\mathrm{Ru}(\mathrm{bpy})_{3}\right]^{3+}} \\
& \mathrm{CNDs}-\mathrm{e}-\rightarrow[\mathrm{CNDs}]+\mathrm{H}^{+}
\end{aligned}
$$

$\left[\operatorname{Ru}(\text { bpy })_{3}\right]^{3+}+[\mathrm{CNDs}]^{\cdot} \rightarrow\left[\operatorname{Ru}(\mathrm{bpy})_{3}\right]^{2+*}$

$\left[\mathrm{Ru}(\mathrm{bpy})_{3}\right]^{2+*} \rightarrow\left[\mathrm{Ru}(\mathrm{bpy})_{3}\right]^{2+}+\mathrm{hv}$

$\left[\mathrm{Ru}(\mathrm{bpy})_{3}\right]^{2+}$ and the CNDs are oxidized at the electrode surface to $\left[\mathrm{Ru}(\mathrm{bpy})_{3}\right]^{3+}$ and the radical [CNDs], respectively. Then, $[\mathrm{CNDs}]$ reduces $\left[\mathrm{Ru}(\mathrm{bpy})_{3}\right]^{3+}$ to $\left[\mathrm{Ru}(\mathrm{bpy})_{3}\right]^{2+*}$, which is unstable and decays to the ground state, emitting a red light.

To investigate why CNDs assist in the anodic ECL of $\left[\mathrm{Ru}(\mathrm{bpy})_{3}\right]^{2+}$, we obtained the UV-visible absorption and photoluminescent emission spectra of solutions of CNDs, $\left[\mathrm{Ru}(\mathrm{bpy})_{3}\right]^{2+}$ and CNDs $+\left[\mathrm{Ru}(\mathrm{bpy})_{3}\right]^{2+}$. The $\mathrm{CNDs}+\left[\mathrm{Ru}(\mathrm{bpy})_{3}\right]^{2+}$ mixture has the same absorption spectrum as $\left[\mathrm{Ru}(\mathrm{bpy})_{3}\right]^{2+}$, with a negligible contribution from the CNDs (Fig. S8A). Something similar occurs in the case of the photoluminescent spectra (Fig. S8B), where $\left[\mathrm{Ru}(\mathrm{bpy})_{3}\right]^{2+}$ and the CNDs maintain their original photoluminescence in the mixture. These results suggest that no reaction takes place between excited-state or ground-state $\mathrm{CNDs}$ and $\left[\mathrm{Ru}(\mathrm{bpy})_{3}\right]^{2+}[35]$. Hence, the light emission must be due to a reaction between the electrogenerated $\left[\mathrm{Ru}(\mathrm{bpy})_{3}\right]^{3+}$ and the CNDs. $\left[\mathrm{Ru}(\mathrm{bpy})_{3}\right]^{2+}$ is identified as the emitter in the mixture; therefore, the CNDs must be the co-reactant. Consequently, the ECL signal of $\left[\mathrm{Ru}(\mathrm{bpy})_{3}\right]^{2+}$ increases on increasing the concentration of CNDs from 40 to $70 \mu \mathrm{M}$ and then level off (Fig. 2B) from the inner filter effect of CNDs [27]. Based on these results, $70 \mu \mathrm{M}$ of CNDs and $7.0 \mathrm{mM}\left[\mathrm{Ru}(\mathrm{bpy})_{3}\right]^{3+}$ were chosen as optimal. Moreover, different $\mathrm{pH}$ values were also studied; the ECL signal of $\left[\mathrm{Ru}(\mathrm{bpy})_{3}\right]^{3+}$ in $0.2 \mathrm{M}$ PB increases with the $\mathrm{pH}$ up to 9.0. However, since biological samples will be used, $\mathrm{pH} 8.0$ was chosen as more adequate. Thus, $70 \mu \mathrm{M}$
Fig. 2 Cyclic voltammograms (A) and ECL signals (B) of a $70 \mu \mathrm{M}$ CNDs (a) or $7 \mathrm{mM}$ $\left[\mathrm{Ru}(\mathrm{bpy})_{3}\right]^{2+}$ in the absence (b) and in the presence of 40 (c), 60 (d), and $70 \mu \mathrm{M}$ CNDs (e) in $0.2 \mathrm{M} \mathrm{PB}, \mathrm{pH} 8.0$ at AuSPE from 0.6 to $+1.20 \mathrm{~V}$ (vs. Ag). Scan rate: $10 \mathrm{mVs}^{-1}$. Inset: scheme of the $\left[\mathrm{Ru}(\mathrm{bpy})_{3}\right]^{2+} /$ CNDs ECL system
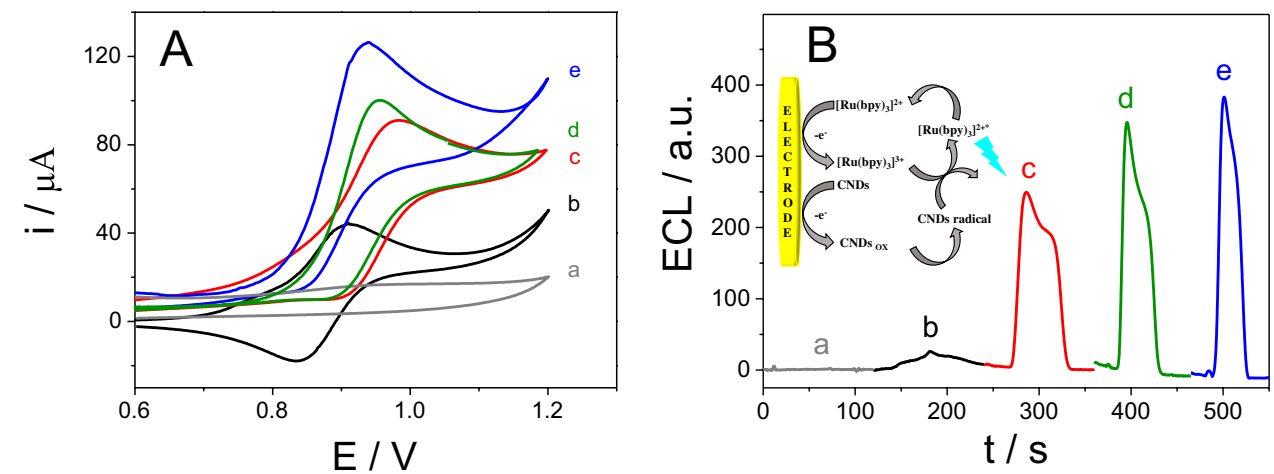
of CNDs and $7.0 \mathrm{mM}\left[\mathrm{Ru}(\mathrm{bpy})_{3}\right]^{3+}$ in $0.2 \mathrm{M} \mathrm{PB}, \mathrm{pH} 8.0$ were chosen as optimal conditions for further experiments.

\section{Electrochemiluminescent miRNA-21biosensor development}

Based on the results described above, using the $\left[\mathrm{Ru}(\mathrm{bpy})_{3}\right]^{2+} /$ CNDs system, we developed an ECL DNA biosensor for the sensitive detection of miRNA-21 (Scheme 1). The $\left[\mathrm{Ru}(\mathrm{bpy})_{3}\right]^{2+} / \mathrm{CNDs}$ ECL system was used to carry out the transduction that allows the biomarker miRNA-21 to be detected and quantified. Scheme 1 shows the different steps taken to design the biosensor.

As explained in the "Experimental" section, the first step is the immobilization of the miRNA-21-SH probe through the chemisorption of thiols on the surface of the AuSPE. In order to obtain a compact and standed-up thiolated probe monolayer, we have performed the immobilization either by direct adsorption of the capture probe alone for long time or employing mercaptohexanol (MCH) in addition to the thiolated probe. As was the case in previous works reported by us [54], best results were obtained when the thiolated probe was directly deposited on the electrode surface for long time $(24 \mathrm{~h})$. With this method, the hybridization effectiveness is higher since we observe the maximum difference between the ECL signal before and after hybridization of the probe with the target miRNA. These results agree well with those previously reported by us and confirm that immobilization time plays an important role in the self-assemble of the capture probe monolayer.

Our method allows also to determine the amount of thiolated oligonucleotide immobilized on the gold surfaces from the coulometry charge associated with the desorptive reduction of the miRNA-21-SH monolayer, as previously described for alkane thiols [55]. The surface coverage was calculated as $2.8 \pm 0.2 \times 10^{-8} \mathrm{~mol}$ oligonucleotide $/ \mathrm{cm}^{2}$.

Next, hybridization of the probe with the complementary analyte sequence (10.0 pM miRNA-21 $1_{\mathrm{C}}$ solution) was performed on the electrode surface. Experimental parameters used, such as buffer, $\mathrm{pH}$, and ionic strength for hybridization, are explained in detail in the "Experimental" section.

Finally, the hybridization event was detected via changes in the signal from the $\left[\mathrm{Ru}(\mathrm{bpy})_{3}\right]^{2+} / \mathrm{CNDs}$ ECL system, after applying a cyclic potential sweep from 0.00 to $+1.30 \mathrm{~V}$ at 10 $\mathrm{mVs}^{-1}$. The resulting ECL signals were plotted as a function of time and compared to the bare electrode ECL signal (a). As can be seen in Fig. 3A, the biosensor response before hybridization (b) is less intense than that observed after hybridization with the fully complementary sequence (c). Specifically, hybridization of the probe produces an increase in the ECL signal of approximately 205 a.u. This can be explained by the formation of a stable helical conformation after hybridization, which enhances the electron-transfer reaction compared to the unstructured single-stranded unit. Therefore, these results demonstrate that the $\left[\mathrm{Ru}(\mathrm{bpy})_{3}\right]^{2+} /$ CNDs system used in the ECL technique can detect hybridization between oligonucleotides.

As a comparison, the same experiments were carried out using the $\left[\mathrm{Ru}(\mathrm{bpy})_{3}\right]^{2+}$ complex alone (in the absence of CNDs). In this case, the ECL spectrum (Fig. 3B) shows that, after modifying the AuSPE with DNA, the signal increases slightly compared to that of the bare electrode. However, the difference in signals from the probe before and after hybridization with the complementary sequence is so small that it is difficult to discriminate between them.

From the above results, we concluded that the synthesized CNDs act as co-reactants for the ECL signal of $\left[\mathrm{Ru}(\mathrm{bpy})_{3}\right]^{2+}$, improving the sensitivity of the method. Furthermore, in the presence of CNDs, there is a significant difference between the signals obtained before and after hybridization, which is essential for the development of a sensitive DNA biosensor.

After verifying the ability of the developed biosensor to detect the specific sequence of miRNA-21 $1_{\mathrm{C}}$, we studied its response to the biomarker at concentrations from $2.34 \mathrm{fM}$ to $100.0 \mathrm{pM}$. The ECL signal increases linearly on increasing the target concentration, as can be seen in Fig. 4A. The plot of biosensor response versus $\log \left[\mathrm{miRNA}-21_{\mathrm{C}}\right.$ ] fits the linear equation $\mathrm{ECL}=29.1 \log \left[\mathrm{miRNA}-21_{\mathrm{C}}\right]+722.3(R=0.9996)$. The method has a sensitivity of 29.1 a.u. $\log \mathrm{pM}^{-1}$, obtained
Fig. 3 Cyclic voltammograms and ECL signal from 0.0 to $+1.3 \mathrm{~V}$ (vs. Ag) of a bare AuSPE (a) and an AuSPE modified with miRNA-21-SH (b) or miRNA-21C/miRNA-21-SH (c) using a $7 \mathrm{mM}$ solution of $\left[\mathrm{Ru}(\mathrm{bpy})_{3}\right]^{2+}$ in $0.2 \mathrm{M} \mathrm{PB}, \mathrm{pH}$ 8.0 , in the presence $(\mathbf{A})$ and in the absence of $70 \mu \mathrm{M}$ CNDs (B). Scan rate: $10 \mathrm{mVs}^{-1}$
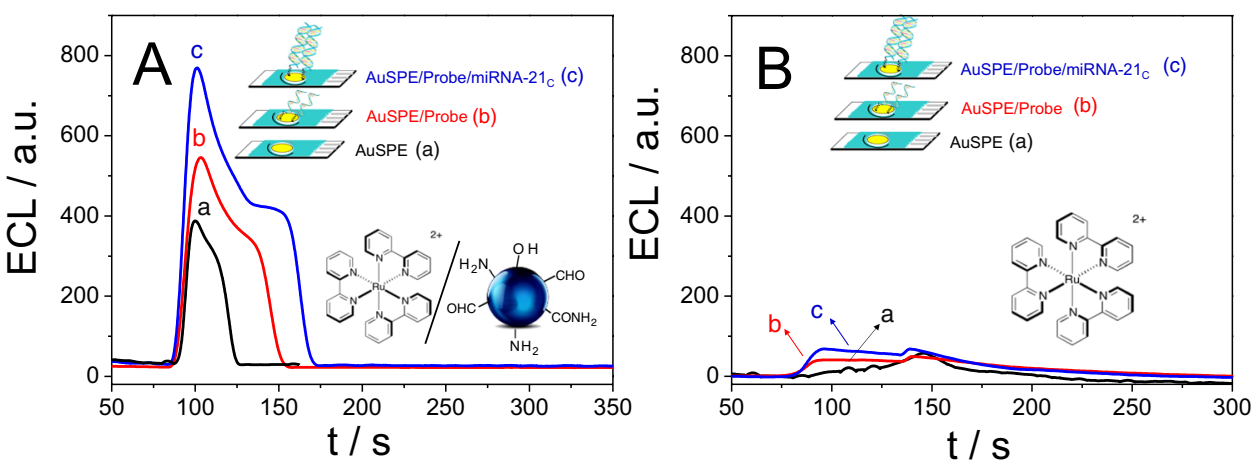


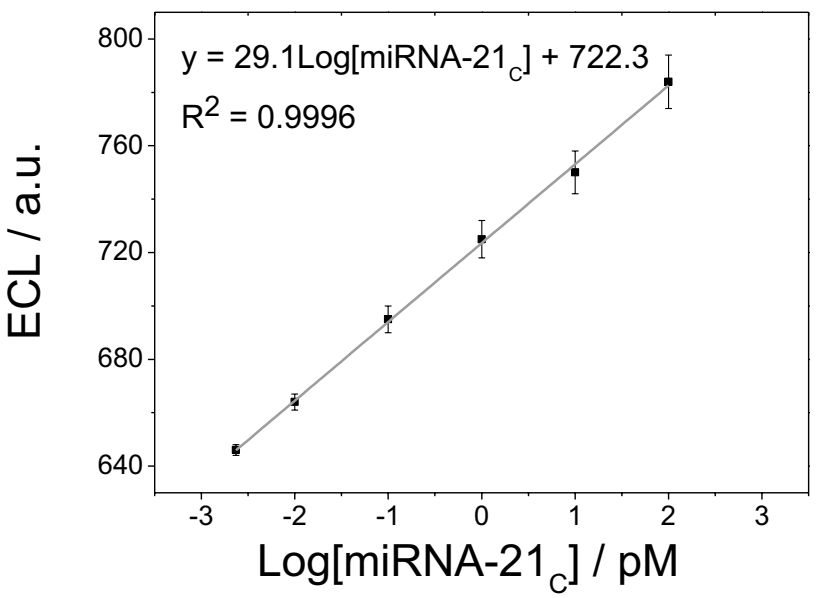

Fig. 4 Calibration plot of the ECL biosensor response vs. miRNA$21_{\mathrm{C}}$ concentration (from $10.0 \mathrm{fM}$ to $100.0 \mathrm{pM}$ )

from the slope of the plot (Fig. 4B). The detection and quantification limits were found to be $0.721(S / N=3)$ and 2.34 $(S / N=10) \mathrm{fM}$, respectively.

Compared to other reported methods for the determination of miRNA-21, the ECL biosensor developed in this work exhibited a lower detection limit (Table 2). It also compares well with previously reported ECL biosensors and has much a wider linear concentration range. Furthermore, the method is simple, without the need for complex approaches or labeling steps.

The selectivity of the ECL biosensor was tested using a non-complementary sequence (miRNA-2 $1_{\mathrm{NC}}$ ) and a single mismatched sequence $\left(\right.$ miRNA- $21_{\mathrm{SM}}$ ). In addition, in order to consider the high homology between miRNA families, the biosensor response to a miRNA144 and miRNA-155 sequence was tested (see Fig. S9). For this purpose, ECL biosensor responses were recorded before and after hybridization with a $100.0 \mathrm{pM}$ solution of a non-complementary $\left(\right.$ miRNA- $21_{\mathrm{NC}}$ ), a single-mismatched (miRNA-2 $1_{\mathrm{SM}}$ ), interferent sequences (miRNA144 and miRNA-155), or a totally complementary sequence (miRNA-21 $1_{C}$ ) used as control. When the probe is hybridized with the complementary sequence, an increase in ECL signal of around $205 \pm 8$ a.u. is observed. However, when hybridization takes place with the mismatched sequence, the increase in the ECL signal is lower (around $125 \pm 7$ a.u.). The hybridization with the single-mismatched target will give a distorted double-helix, causing a decrease in the ECL signal. In the case of the noncomplementary and interferent sequences (miRNA-144 and miRNA-155), a response very similar to that of the probe is observed. This result suggests that no hybridization process is taking place; the small increase observed is probably due to a nonspecific adsorption. The reproducibility of the method was estimated from five devices prepared using the same protocol. In all cases, the relative standard deviation (RSD) of the ECL signal was found to be less than 7\%. Moreover, the biosensor can detect the target miRNA- $21_{\mathrm{C}}$ over a period of 1 month.

\section{miRNA-21 determination in spiked human serum samples}

Finally, we evaluated the applicability of the biosensor by determining miRNA-21 in human serum samples using an external calibration method, as described in detail the "Experimental" section. ECL measurements gave an average value of 723.8 a.u. From the calibration plot, the miRNA-21 concentration in the spiked serum sample (final miRNA-21 concentration of $1.00 \mathrm{pM}$ ) was found to be $1.13 \mathrm{pM}$ with a recovery of $113 \%$ and a RSD of $4 \%$. This result demonstrates that the proposed biosensor can be used for practical applications and has great potential as an alternative to the classical methods for detecting miRNA-21 in human serum samples.

\section{miRNA-21 determination in serum samples from heart failure patients}

Based on the great interest of having simple methodologies for rapid biomarker detection and considering the good results obtained with the developed biosensor in spiked human serum samples, we take a step forward and applied our methodology to detect miRNA-21 directly in serum
Table 2 Analytical parameters of different methods for miRNA-21 detection

\begin{tabular}{llll}
\hline Detection technique & LOD (fM) & Linear range (fM to pM) & Ref \\
\hline Colorimetric & $3.2 \times 10^{6}$ & $10 \times 10^{6}$ to $0.9810^{6}$ & {$[56]$} \\
Fluorescent spectroscopy & $4.2 \times 10^{3}$ & $10 \times 10^{3}$ to $2.0 \times 10^{3}$ & {$[57]$} \\
Electrochemical & 30 & 100 to $2 \times 10^{3}$ & {$[58]$} \\
Electrochemical & 1.5 & 5 to $2 \times 10^{3}$ & {$[59]$} \\
Organic electrochemical transistors & $2 \times 10^{3}$ & $5 \times 10^{3}$ to $20 \times 10^{3}$ & {$[60]$} \\
ECL & 0.65 & 1.0 to 100 & {$[44]$} \\
ECL & 0.03 & 0.1 to 10 & {$[41]$} \\
ECL & 2.78 & 0.10 to 100 & {$[39]$} \\
ECL & 0.721 & 2.34 to 100 & This work \\
\hline
\end{tabular}


samples from heart failure patients, provided and qRT-PCR checked by IRICYS (Instituto Ramón y Cajal de Investigación Sanitaria), as we described in detail in the "Experimental" section. Two different types of serums were analyzed (serum 1 and serum 2, latest used as control). It is worth to note that in the case of qRT-PCR method, it is necessary to include a RNA extraction step before qRT-PCR analysis and the whole process requires long time of analysis. The developed ECL biosensors only require a denaturation step (see the "Experimental" section) before the analysis. The ECL biosensor response to the serum 1 (Fig. S10) shows a signal of $724 \pm 10$ a.u. compared to the signal of the probe ( $575 \pm 6$ a.u.). The concentration was estimated using the calibration plot $\left(E C L=29.1 \log \left[\right.\right.$ miRNA- $\left.\left.21_{C}\right]+722.3\right)$. A concentration of $1.14 \mathrm{pM}$ was obtained. As we describe in the "Experimental" section, serum 2 was used as control. In this case, the biosensor response to serum 2 shows a signal of $600 \pm 8$ a.u., suggesting that the miRNA-21 concentration is extremely low and no miRNA-21 overexpression is observed. These results agree well with those obtained by qRT-PCR which renders $\mathrm{Ct}=23$ in serum sample 1 and $\mathrm{Ct}=31$ in serum sample 2. Normalizer and controls (miR103, UniSp2, and cel 39) did not show differences in $\mathrm{Ct}$ between both samples.

Based on the above results, we can conclude that the biosensor developed can detect a miRNA-21 sequence directly in clinical human serum samples from heart failure patients without any previous amplification process. It is a simple, sensitive, fast, and scalable tool for detecting this biomarker, allowing early detection of the disease and increasing the probability of patient survival.

\section{Conclusions}

CNDs have been synthesized through an environment friendly chemistry-based procedure using tiger nut milk as a natural precursor and have been demonstrated to be excellent co-reactants for the development of an improved $\left[\mathrm{Ru}(\mathrm{bpy})_{3}\right]^{2+}$ ECL DNA biosensor. The $\left[\mathrm{Ru}(\mathrm{bpy})_{3}\right]^{2+} /$ CNDs system was used for the first time to detect the specific sequence of a biomarker associated with breast cancer, miRNA-21. Electrochemical studies have confirmed that CNDs catalyze the oxidation of the $\left[\mathrm{Ru}(\mathrm{bpy})_{3}\right]^{2+}$ complex on DNA-modified AuSPEs, improving the ECL signal. The $\left[\mathrm{Ru}(\mathrm{bpy})_{3}\right]^{2+} / \mathrm{CNDs}$ system is highly promising for bioanalysis, as exemplified in this work by the detection of a cancer biomarker in spiked human serum samples as well in clinical serum samples from heart failure patients without any previous amplification process. This work has opened new approaches for the synthesis of CNDs and has deepened and broadened our knowledge, as well as the application of these nanomaterials for biosensing, which will be beneficial for further development based on CNDs.

Supplementary Information The online version contains supplementary material available at https://doi.org/10.1007/s00604-021-05038-y.

Acknowledgements The authors acknowledge Confocal Microscopy and Flow Cytometry Services of CBMSO and "Ayudas para el fomento de la investigación en estudios de Máster" provided by the Universidad Autónoma de Madrid. Serum samples from patients included in this study were provided by the BioBank Hospital Ramón y Cajal-IRYCIS (National Registry of Biobanks B.0000678), integrated in the Biobanks and Biomodels Platform of the ISCIII (PT20/00045), and they were processed following standard operating procedures with the appropriate approval of the Ethical and Scientific Committees.

Funding Open Access funding provided thanks to the CRUE-CSIC agreement with Springer Nature. This study is funded by the Comunidad Autónoma de Madrid (Spain) projects (TRANSNANOAVANSENS, S2018/NMT-4349, CAM/B2017/BMD-3686) and Ministerio de Economía, Industria y Competitividad (Spanish Government) projects: CTQ2015-71955-REDT (ELECTROBIONET), CTQ2014-53334-C21-R and PID2020-116728RB-I00.

\section{Declarations}

Conflict of interest The authors declare no competing interests.

Open Access This article is licensed under a Creative Commons Attribution 4.0 International License, which permits use, sharing, adaptation, distribution and reproduction in any medium or format, as long as you give appropriate credit to the original author(s) and the source, provide a link to the Creative Commons licence, and indicate if changes were made. The images or other third party material in this article are included in the article's Creative Commons licence, unless indicated otherwise in a credit line to the material. If material is not included in the article's Creative Commons licence and your intended use is not permitted by statutory regulation or exceeds the permitted use, you will need to obtain permission directly from the copyright holder. To view a copy of this licence, visit http://creativecommons.org/licenses/by/4.0/.

\section{References}

1. Dong $\mathrm{Y}$ et al (2012) Polyamine-functionalized carbon quantum dots for chemical sensing. Carbon 50(8):2810-2815

2. Prato M (1997) [60]Fullerene chemistry for materials science applications. J Mater Chem 7(7):1097-1109

3. Tasis D et al (2006) Chemistry of carbon nanotubes. Chem Rev 106(3):1105-1136

4. Baker SN, Baker GA (2010) Luminescent carbon nanodots: emergent nanolights. Angew Chem Int Ed 49(38):6726-6744

5. Cao L et al (2007) Carbon dots for multiphoton bioimaging. J Am Chem Soc 129(37):11318-11319

6. Pirsaheb M, Mohammadi S, Salimi A (2019) Current advances of carbon dots based biosensors for tumor marker detection, cancer cells analysis and bioimaging. TrAC, Trends Anal Chem 115:83-99

7. Valizadeh A et al (2012) Quantum dots: synthesis, bioapplications, and toxicity. Nanoscale Res Lett 7(1):480

8. Bahari D et al (2020) A self-enhanced ECL-RET immunosensor for the detection of CA19-9 antigen based on Ru(bpy)2(phen$\mathrm{NH} 2) 2+-$ amine-rich nitrogen-doped carbon nanodots as probe 
and graphene oxide grafted hyperbranched aromatic polyamide as platform. Anal Chim Acta 1132:55-65

9. Wu D et al (2016) Label-free electrochemiluminescent immunosensor for detection of prostate specific antigen based on aminated graphene quantum dots and carboxyl graphene quantum dots. Sci Rep 6(1):20511

10. Guerrero-Esteban T et al (2021) Sensitive glyphosate electrochemiluminescence immunosensor based on electrografted carbon nanodots. Sens Actuators B Chem 330:129389

11. Richter MM (2004) Electrochemiluminescence (ECL). Chem Rev 104(6):3003-3036

12. Valenti $G$ et al (2018) Electrogenerated chemiluminescence from metal complexes-based nanoparticles for highly sensitive sensors applications. Coord Chem Rev 367:65-81

13. Sojic $\mathrm{N}$ et al (2017) Applications of electrogenerated chemiluminescence in analytical chemistry. In: Miomandre F, Audebert P (eds) luminescence in electrochemistry: applications in analytical chemistry, physics and biology. Springer International Publishing, Cham, pp 257-291

14. Miao W (2008) Electrogenerated chemiluminescence and its biorelated applications. Chem Rev 108(7):2506-2553

15. Martínez-Periñán E et al. (2010) Electrochemiluminescence biosensors using screen-printed electrodes. Biosensors 10(9)

16. Xu X-H, Bard AJ (1995) Immobilization and hybridization of DNA on an aluminum(III) alkanebisphosphonate thin film with electrogenerated chemiluminescent detection. J Am Chem Soc 117(9):2627-2631

17. Miao W (2007) 13 - Electrogenerated chemiluminescence. In: Zoski CG (ed) Handbook of Electrochemistry. Elsevier, Amsterdam, pp 541-590

18. Miao W, Bard AJ (2004) Electrogenerated chemiluminescence. 77. DNA hybridization detection at high amplification with $[\mathrm{Ru}(\mathrm{bpy}) 3] 2+-$ containing microspheres. Anal Chem 76(18):5379-5386

19. Li Y et al (2007) Ultrasensitive electrogenerated chemiluminescence detection of DNA hybridization using carbon-nanotubes loaded with tris(2,2'-bipyridyl) ruthenium derivative tags. Talanta 72(5):1704-1709

20. Miao W, Bard AJ (2003) Electrogenerated chemiluminescence. 72. Determination of immobilized DNA and C-reactive protein on $\mathrm{Au}(111)$ electrodes using tris(2,2'-bipyridyl)ruthenium(II) labels. Anal Chem 75(21):5825-5834

21. Bertolino $\mathrm{C}$ et al (2005) A monolithic silicon based integrated signal generation and detection system for monitoring DNA hybridisation. Biosens Bioelectron 21(4):565-573

22. Spehar-Deleze A-M et al (2006) Electrochemiluminescent hybridization chip with electric field aided mismatch discrimination. Biosens Bioelectron 22(5):722-729

23. Firrao G (2005) Detection of DNA/DNA hybridization by electrogenerated chemiluminescence. Int J Environ Anal Chem 85(9-11):609-612

24. Spehar A-M et al (2004) The quenching of electrochemiluminescence upon oligonucleotide hybridization. Luminescence 19(5):287-295

25. Dong Y et al (2010) Extraction of electrochemiluminescent oxidized carbon quantum dots from activated carbon. Chem Mater 22(21):5895-5899

26. Zheng L et al (2009) Electrochemiluminescence of water-soluble carbon nanocrystals released electrochemically from graphite. J Am Chem Soc 131(13):4564-4565

27. Long Y-M et al (2014) Revealing carbon nanodots as coreactants of the anodic electrochemiluminescence of Ru(bpy)32+. Anal Chem 86(15):7224-7228

28. Mittal S et al (2017) Biosensors for breast cancer diagnosis: a review of bioreceptors, biotransducers and signal amplification strategies. Biosens Bioelectron 88:217-231
29. Nouraee N and Mowla SJ (2015) miRNA therapeutics in cardiovascular diseases: promises and problems. Front Genet 6(232)

30. Roy S et al (2009) MicroRNA expression in response to murine myocardial infarction: miR-21 regulates fibroblast metalloprotease- 2 via phosphatase and tensin homologue. Cardiovasc Res 82(1):21-29

31. Thum $\mathrm{T}$ et al (2008) MicroRNA-21 contributes to myocardial disease by stimulating MAP kinase signalling in fibroblasts. Nature 456(7224):980-984

32. Tijsen AJ, Pinto YM, Creemers EE (2011) Non-cardiomyocyte microRNAs in heart failure. Cardiovasc Res 93(4):573-582

33. Yin B-C, Liu Y-Q, Ye B-C (2012) One-step, multiplexed fluorescence detection of microRNAs based on duplex-specific nuclease signal amplification. J Am Chem Soc 134(11):5064-5067

34. Miao P et al (2018) Electrochemical detection of miRNA combining T7 exonuclease-assisted cascade signal amplification and DNA-templated copper nanoparticles. Anal Chem 90(18):11154-11160

35. White AK et al (2011) High-throughput microfluidic single-cell RT-qPCR. Proc Natl Acad Sci 108(34):13999-14004

36. Zhang $\mathrm{S}$ et al (2016) Multiplex miRNA assay using lanthanidetagged probes and the duplex-specific nuclease amplification strategy. Chem Commun 52(99):14310-14313

37. Xia Y et al (2018) A ratiometric fluorescent bioprobe based on carbon dots and acridone derivate for signal amplification detection exosomal microRNA. Anal Chem 90(15):8969-8976

38. Laurenti $\mathrm{M}$ et al (2016) Enhancement of the upconversion emission by visible-to-near-infrared fluorescent graphene quantum dots for miRNA detection. ACS Appl Mater Interfaces $8(20): 12644-12651$

39. Liu G et al (2020) Bright carbon nanodots for miRNA diagnostics coupled with concatenated hybridization chain reaction. Chem Commun 56(8):1175-1178

40. Cao X et al (2020) Calcium ion assisted fluorescence determination of microRNA-167 using carbon dots-labeled probe DNA and polydopamine-coated $\mathrm{Fe} 3 \mathrm{O} 4$ nanoparticles. Microchim Acta 187(4):212

41. Zhong $X$ et al (2020) Synthesizing anode electrochemiluminescent self-catalyzed carbon dots-based nanocomposites and its application in sensitive ECL biosensor for microRNA detection. Sens Actuators B Chem 305:127490

42. Ye J et al (2019) Highly luminescent and self-enhanced electrochemiluminescence of Tris(bipyridine) ruthenium(II) nanohybrid and its sensing application for label-free detection of MicroRNA. Anal Chem 91(20): 13237-13243

43. Xu Z et al (2017) Ultrasensitive electrochemiluminescence biosensor for MicroRNA detection by 3D DNA walking machine based target conversion and distance-controllable signal quenching and enhancing. Anal Chem 89(16):8282-8287

44. Zhang $Y$ et al (2020) Electrochemiluminescence biosensor for miRNA-21 based on toehold-mediated strand displacement amplification with $\mathrm{Ru}($ phen) $32+$ loaded DNA nanoclews as signal tags. Biosens Bioelectro 147:111789

45. Liao $\mathrm{N}$ et al (2020) DNA structure transition-induced affinity switch for biosensing based on the strong electrochemiluminescence platform from organic microcrystals. Anal Chem 92(5):3940-3948

46. Li J et al (2020) Ultrasensitive electrochemiluminescence biosensing platform for miRNA-21 and MUC1 detection based on dual catalytic hairpin assembly. Anal Chim Acta 1105:87-94

47. Strauss $\mathrm{V}$ et al (2016) Assigning electronic states in carbon nanodots. Adv Func Mater 26(44):7975-7985

48. Jin B, Ji X, Nakamura T (2004) Voltammetric study of interaction of Co(phen)33+ with DNA at gold nanoparticle self-assembly electrode. Electrochim Acta 50(4):1049-1055 
49. Shen $\mathbf{J}$ et al (2017) Facile synthesis of fluorescence carbon dots from sweet potato for $\mathrm{Fe} 3+$ sensing and cell imaging. Mater Sci Eng, C 76:856-864

50. Yang X et al (2014) Novel and green synthesis of high-fluorescent carbon dots originated from honey for sensing and imaging. Biosens Bioelectron 60:292-298

51. Prasannan A, Imae T (2013) One-pot synthesis of fluorescent carbon dots from orange waste peels. Ind Eng Chem Res 52(44):15673-15678

52. Mehta VN et al (2015) One-step hydrothermal approach to fabricate carbon dots from apple juice for imaging of mycobacterium and fungal cells. Sens Actuators, B Chem 213:434-443

53. Carrara $S$ et al (2017) Inside back cover: amine-rich nitrogendoped carbon nanodots as a platform for self-enhancing electrochemiluminescence. Angew Chem Int Ed 56(17) 4891-4891

54. García T et al (2010) Disposable sensors for rapid screening of mutated genes. Anal Bioanal Chem 398(3):1385-1393

55. Widrig CA, Chung C, Porter MD (1991) The electrochemical desorption of $\mathrm{n}$-alkanethiol monolayers from polycrystalline $\mathrm{Au}$ and $\mathrm{Ag}$ electrodes. J Electroanal Chem Interfacial Electrochem 310(1):335-359

56. Zhao $\mathrm{H}$ et al (2016) A visible and label-free colorimetric sensor for miRNA-21 detection based on peroxidase-like activity of graphene/gold-nanoparticle hybrids. Anal Methods 8(9):2005-2012

57. $\mathrm{Xu} \mathrm{S}$ et al (2018) Polydopamine nanosphere/gold nanocluster ( $\mathrm{Au}$ $\mathrm{NC}$ )-based nanoplatform for dual color simultaneous detection of multiple tumor-related MicroRNAs with DNase-I-assisted target recycling amplification. Anal Chem 90(6):4039-4045

58. Yao J et al (2017) An enzyme free electrochemical biosensor for sensitive detection of miRNA with a high discrimination factor by coupling the strand displacement reaction and catalytic hairpin assembly recycling. Analyst 142(21):4116-4123

59. Shen $Z$ et al (2020) Highly sensitive and simultaneous detection of microRNAs in serum using stir-bar assisted magnetic DNA nanospheres-encoded probes. Biosens Bioelectron 148:111831

60. Peng J et al (2018) An organic electrochemical transistor for determination of microRNA21 using gold nanoparticles and a capture DNA probe. Microchim Acta 185(9):408

Publisher's note Springer Nature remains neutral with regard to jurisdictional claims in published maps and institutional affiliations. 